\title{
DEM of triaxial tests on crushable sand
}

\author{
J. P. de Bono • G. R. McDowell
}

Received: 29 January 2014 / Published online: 29 April 2014

(C) Springer-Verlag Berlin Heidelberg 2014

\begin{abstract}
This paper presents simulations of high-pressure triaxial shear tests on a crushable sand. The discrete element method is used, featuring a large number of particles and avoiding the use of agglomerates. The triaxial model features a flexible membrane, therefore allowing realistic deformation, and a simple breakage mechanism is implemented using the octahedral shear stress induced in the particles. The simulations show that particle crushing is essential to replicate the realistic behaviour of sand (in particular the volumetric contraction) in high-pressure shear tests. The general effects of crushing during shear are explored, including its effects on critical states, and the influence of particle strength and confining pressure on the degree of crushing are discussed.
\end{abstract}

Keywords Discrete element method - DEM - Particle crushing $\cdot$ Triaxial

\section{Introduction}

The macroscopic behaviour of a soil, in particular the strength and strain is largely affected by the degree of particle crushing. This is particularly true for triaxial shear tests, where the breakage of particles decreases the rate of dilation [1]. The degree of particle crushing is influenced by a number of factors (including size, shape, mineralogy, water condition, etc.), principally the strength of the grains and the effective stress state. As such, the effects of particle crushing are greatest at high pressures (e.g.[2]).

In recent years, crushable particles have been modelled using the discrete element method (DEM) either by using

J. P. de Bono · G. R. McDowell ( $₫)$

University of Nottingham, Nottingham, UK

e-mail: glenn.mcdowell@ nottingham.ac.uk agglomerates ('grains' consisting of smaller elementary spheres, bonded together), or by replacing 'broken' particles with smaller, self-similar fragments. Using the former method, Bolton et al. [3] showed that crushable particles are necessary for achieving realistic levels of volumetric contraction when modelling triaxial shear tests on granular soils.

However, one problem with using agglomerates is the large number of elementary particles required, which severely limits the overall number of grains that can be used; Bolton et al. [3] used just 389 agglomerates, consisting of less than 50 spheres each; Lim and McDowell [4] showed that each agglomerate ideally should comprise at least 500 spheres to correct capture the size effect on strength.

This paper aims to show that it is possible to simulate the phenomenon of particle crushing in high-pressure triaxial tests on sand, using DEM with a large number of particles, by using a triaxial model which features a flexible membrane allowing accurate deformation [5], and incorporating a simple breakage mechanism which replaces broken sand particles with new, smaller grains [6]. The following work aims to confirm that particle crushing is essential to replicate realistic behaviour of sand in realistic triaxial conditions, as well as to investigate the nature of crushing and its effects on the critical state behaviour. The general effects of particle crushing during shear are explored, as well as the influence of particle strength and confining pressure on the degree of crushing.

\section{Triaxial model}

\subsection{Specimen}

The sand particles are modelled using spheres; the triaxial sample used is cylindrical, with a height of $100 \mathrm{~mm}$ and a 
diameter of $50 \mathrm{~mm}$. The Hertz-Mindlin contact model [7] is used-the sand particles are given a Poisson's ratio, $v=0.25$ and a shear modulus, $G=28 \mathrm{GPa}$; typical values for quartz. The ability of the Hertzian contact model to capture the nonlinear stiffness of spheres offers more realism than linear springs, and was considered appropriate due to the importance of induced particle stress in the simulations. The initial specimen is mono-disperse, consisting of 3,350 particles of uniform size $d_{0}=4 \mathrm{~mm}$, and is generated using the radii expansion method [7] to give an initial voids ratio $e_{0}=0.75$. The initial diameter determines the number particles generated, and although a quantity of 3,350 may be considered small, it is greater than the amount used in similar simulations of crushable soil (e.g. [3,8]). Furthermore, the particles can break an unlimited number of times, giving a higher 'breakage capacity' than the agglomerates used in the aforementioned studies. This relatively large initial diameter was chosen for computational efficiency, using a smaller initial diameter would not only result in a greater number of sand particles, but also require a much larger number of membrane particles, which are required to possess a smaller diameter. Although the initial monodispersity is somewhat unrealistic, this was chosen to emphasise the incidence of crushing; the following work serves as an investigation into the effects of crushing during shear, rather than a direct calibration against physical tests.

\subsection{Membrane}

The flexible membrane used is the same as described in de Bono et al. [5], full details of which won't be repeated, the only principal difference being that the Hertz-Mindlin contact model is used in the current simulations. To summarise, the membrane particles were given artificially high stiffness both to prevent them from penetrating the specimen and to keep them aligned, with a system in place to remove the additional hoop tension which resulted. The membrane particles were bonded using contact bonds, which were vanishingly small and transmitted no moments. In this work, due to the different contact model used, membrane particles are ascribed a Poisson's ratio $v=0.5$ (typical for rubber) and given an arbitrarily high shear modulus of $G=1 \mathrm{MPa}$ to prevent penetration. The membrane particles are bonded with parallel bonds (Itasca 2005) instead of contact bonds, the diameter of which are $10^{-10}$ times smaller than the particles. The parallel bonds are given stiffnesses (stress per displacement) of $1 \times 10^{40} \mathrm{~Pa} / \mathrm{m}$; sufficient to keep the membrane particles aligned, and arbitrarily high strengths to avoid perforation (details given in Table 1). The excess hoop tension is alleviated by allowing the membrane particles to expand, details of which are in de Bono et al. [5].
Table 1 Summary of DEM parameters for triaxial model

\begin{tabular}{|c|c|}
\hline \multicolumn{2}{|l|}{ Triaxial sample properties } \\
\hline Size: height $\times$ diameter $(\mathrm{mm})$ & $100 \times 50$ \\
\hline No. of particles & 3,350 \\
\hline Particle friction coefficient & 0.5 \\
\hline Contact model & Hertz-Mindlin \\
\hline Shear modulus, $G(\mathrm{GPa})$ & 28 \\
\hline Poisson's ratio, $v$ & 0.25 \\
\hline Density $\left(\mathrm{kg} / \mathrm{m}^{3}\right)$ & 2,650 \\
\hline Coefficient of uniformity, $C_{\mathrm{u}}$ & 1.0 \\
\hline Particle diameter, $d_{0}(\mathrm{~mm})$ & 4.00 \\
\hline Voids ratio, $e_{0}$ & 0.75 \\
\hline $37 \%$ Strength, $q_{0}(\mathrm{MPa})$ & $20.0\left(\right.$ for $\left.d=d_{0}\right)$ \\
\hline Weibull modulus, $m$ & 3.3 \\
\hline Wall friction coefficient & 0 \\
\hline \multicolumn{2}{|l|}{ Triaxial membrane properties } \\
\hline No. of particles & 3,224 \\
\hline Friction coefficient & 0 \\
\hline Contact model & Hertz-Mindlin \\
\hline Shear modulus, $G(\mathrm{GPa})$ & 1 \\
\hline Poisson's ratio, $v$ & 0.5 \\
\hline Density $\left(\mathrm{kg} / \mathrm{m}^{3}\right)$ & 1,000 \\
\hline Particle diameter, $d_{0}(\mathrm{~mm})$ & 2.66 \\
\hline Parallel bond diameter (mm) & $2.66 \times 10^{-10}$ \\
\hline Parallel bond stiffness $(\mathrm{Pa} / \mathrm{m})$ & $1 \times 10^{40}$ \\
\hline Parallel bond strength $(\mathrm{Pa})$ & $1 \times 10^{30}$ \\
\hline
\end{tabular}

\subsection{Particle crushing}

Crushing has generally been modelled using DEM via two alternatives: replacing 'breaking' grains with new, smaller fragments, generally in two-dimensions [9-13] or by using three-dimensional agglomerates $[3,8,14,15]$. In the latter method, no consideration was given to the complex distribution of loads on each particle at its multiple contacts; however, McDowell and de Bono [6] allowed particles to fracture without the use of agglomerates by considering the stresses induced in a particle due to the multiple contacts. Their work showed macro-scale agreement with both experimental results and theoretical predictions, as well as microscale agreement in the form of fractal geometry. The same breakage mechanism and criteria are used in the following simulations, in which each particle is allowed to split into two new fragments, when the value of induced particle stress is found to be greater or equal to its strength. The new sphere fragments overlap enough to be contained within the bounding parent sphere, with the axis joining the new spheres aligned in the direction of the minor principal stress (Fig. 1). The total volume of the new spheres is equal to that of the original parent sphere, obeying conservation of mass. 


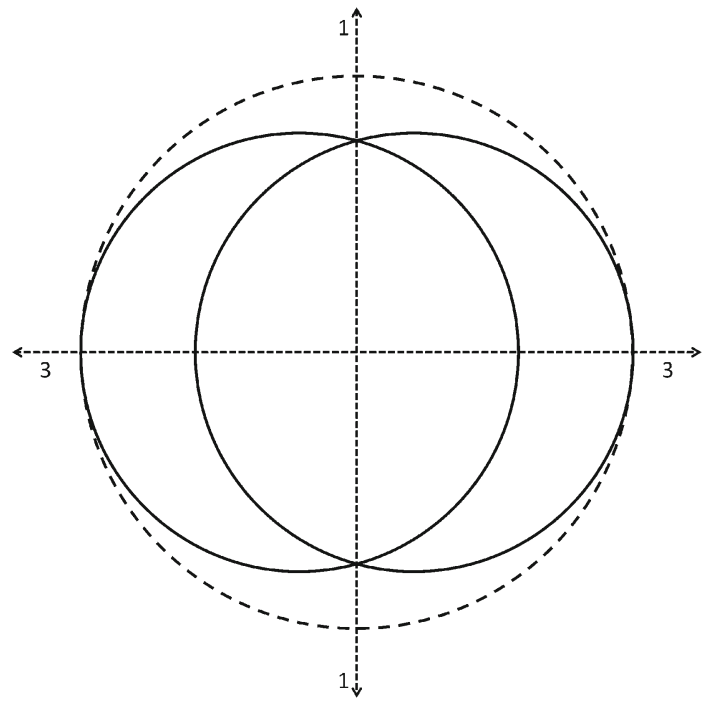

Fig. 1 Equal diametral splitting mechanism

This produces local pressure spikes during breakage; however the fragments move along the direction of the minor principal stress for the original particle, just as would occur for a single particle crushed between platens. Although conservation of energy is not observed in this case, the goal is to achieve an effective breakage mechanism as simple and realistic as possible. As several of the above authors (e.g. [9]) have conjectured, it is not possible to simulate perfectly realistic fracture using self-similar fragments; however it is not the purpose of this study to resolve this problem, but rather to adopt the best approach to investigate particle crushing in high-pressure triaxial tests.

McDowell and de Bono [6] showed that the octahedral shear stress, $q$ within a particle, given by:

$q=\frac{1}{3}\left[\left(\sigma_{1}-\sigma_{2}\right)^{2}+\left(\sigma_{2}-\sigma_{3}\right)^{2}+\left(\sigma_{1}-\sigma_{3}\right)^{2}\right]^{1 / 2}$

could be used to determine whether fracture should occur or not, calculated from the average principal stresses $\left(\sigma_{1}, \sigma_{2}, \sigma_{3}\right)$ within a particle, which are in turn calculated from the contact forces acting on the particle.

Jaeger [16] proposed the tensile strength of grains could be measured by diametral compression between platens as $\sigma=F / d^{2}$. In PFC3D, for a sphere of size $d$ compressed between two walls exerting force $F$, the value of induced octahedral shear stress, $q$, was found to be:

$q=0.9 \frac{F}{d^{2}}$

and so proportional to the assumed induced stress in particle crushing tests. The octahedral shear stress was deemed an appropriate means by which to determine fracture, as it takes into account multiple contacts and complex distribution of loads while avoiding the use of agglomerates.
By crushing individual sand particles, McDowell et al. $[17,18]$ showed that the stresses at failure for a given particle size satisfied a Weibull distribution of strengths (with same variation regardless of size). The mean strength $\sigma_{\mathrm{m}}$ of the particles was related to size by $\sigma_{\mathrm{m}}=d^{-b}$ (where $b$ describes the size-hardening law). Hence McDowell and de Bono [6] assumed a particle would break when the octahedral shear stress was greater than or equal to its strength, where the strengths of the particles satisfy a Weibull distribution of $q$ values. The strengths were related to size by:

$q_{0} \propto d^{-b}$

which, assuming that the Weibull size effect is applicable to soil particles [18], leads to:

$q_{0} \propto d^{-3 / m}$

where $q_{0}$ is the characteristic strength, and is a value of the distribution such that $37 \%$ (i.e. exp[-1]) of random strengths are greater-and is related to the mean; $m$ is the modulus, which is directly related to the coefficient of variation.

In the following triaxial simulations, the top platen is accelerated downwards, then decelerated and stopped after an increase in axial strain of $0.01 \%$; i.e. the simulations are strain controlled. After each increment, the particles are checked and allowed to break; any broken particles are replaced with new fragments. A number of timesteps (inversely proportional to the size of the numerical timestep) are then completed, over the course of which no particles may break; this is to allow the artificial energy from new overlapping particles to dissipate. These two processes are repeated (allowing fragments to break multiple times if necessary) until no further breakages occur; after which the next strain increment is applied. Particle breakage is determined using the octahedral shear stress according to Eq. (1). The initial particles $(d=4) \mathrm{mm}$ have random strengths from a Weibull distribution, defined by the Weibull parameters $q_{0}=20 \mathrm{MPa}$ and $m=3.3$. These values are determined from the data obtained from single particle crushing tests by McDowell [19]. The size-hardening law is governed by Eq. (4), which is used to attribute random strengths to new fragments. The details of the specimen and membrane used in the following simulations are given in Table 1, unless otherwise stated.

The triaxial test variables monitored and recorded during the simulations are the deviatoric stress, $q$, the axial strain, $\varepsilon_{a}$, and volumetric strain, $\varepsilon_{\mathrm{v}}$. The deviatoric stress is measured as the difference between the axial stress (the major principal stress) and confining pressure (minor principal stress), where the axial stress is obtained from the average stress acting on the top and bottom platens. The volumetric strain is calculated from the current and original volumes of the sample, which are obtained from the locations of the membrane particles [5]. 


\section{Crushing and particle strength}

To demonstrate the effects of particle crushing, simulations with and without the possibility of crushing are presented. Figure 2 presents the triaxial results of simulations of three mono-disperse samples $\left(d_{0}=4 \mathrm{~mm}\right)$, with initial voids ratios of 0.75 , subjected to shearing under $1 \mathrm{MPa}$ confining pressure. The results comprise one simulation with unbreakable particles, and two with particles that can breakdemonstrating the influence of particle strength on crushing. Of the breakable simulations, one has initial (for $d_{0}=4 \mathrm{~mm}$ ) particle strengths as above for silica sand, i.e. $q_{0}=20 \mathrm{MPa}$, the other has strengths half the magnitude, i.e. $q_{0}=10 \mathrm{MPa}$. The sand particle strengths in both of these crushable simulations have the same Weibull modulus, $m=3.3$, and follow the same hardening law expressed in (4).

There is no major difference visible between the unbreakable simulation and the material with the stronger particles $\left(q_{0}=20 \mathrm{MPa}\right)$ in the stress-strain responses. They demonstrate almost identical deviatoric stress curves; there is almost no difference in the volumetric strain curves either, although it could be interpreted that the material with no particle breakage is exhibiting slightly higher dilatancy at the end
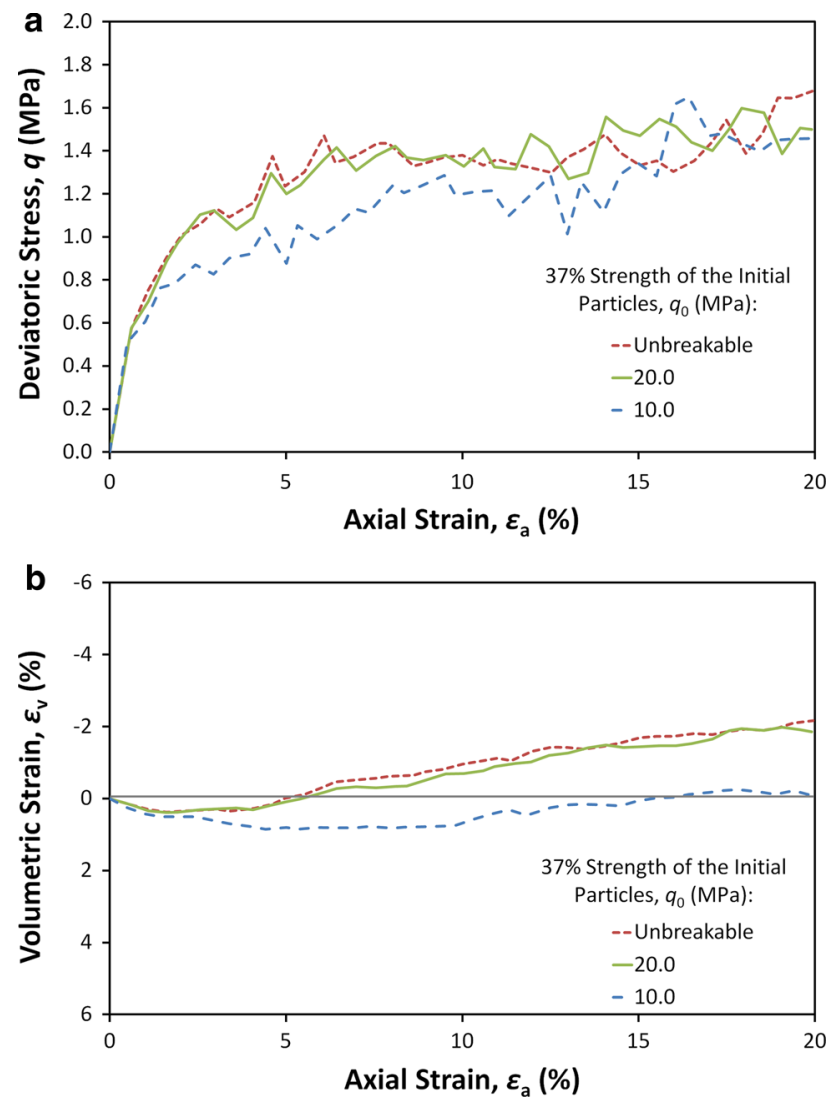

Fig. 2 Triaxial simulation results of an unbreakable sample, and two breakable materials with different particle strengths: deviatoric stress response (a) and volumetric behaviour (b) of the test. If smaller (and therefore stronger) initial particles were used, e.g. $d_{0}=1 \mathrm{~mm}$, even less difference would be expected between the behaviour of two equivalent crushable and uncrushable simulations-as the more numerous, smaller particles would be stronger and exhibit less breakage. For the weaker crushable material, the very initial part of the deviatoric stress response (usually considered elastic) is the same until an axial strain of approximately $1 \%$; after which this stress curve becomes less steep. However, this curve appears to lead to a value of ultimate strength similar to the stronger breakable material. This would suggest that the two breakable soils (and indeed the unbreakable material) have the same critical state parameter $M$, meaning the frictional resistance is the same; the same deviatoric stress is required to keep the soil flowing continuously at a critical state. This may be expected, as the two crushable soils-although consisting of different quantities and sizes of particles after crushing - have the same particle friction coefficient and particle shape. The simulations have slightly fluctuating deviatoric stress curves, which is a result of the relatively small overall number of particles. This effect is exacerbated in the simulations with breakable particles, as will be shown and discussed later.

Figure $2 \mathrm{~b}$ shows that the breakable material with weaker particles $\left(q_{0}=10 \mathrm{MPa}\right)$ undergoes less dilation compared to the unbreakable simulation; this is not surprising and is the same observation as that made by Bolton et al. [3], who subjected breakable and unbreakable agglomerates to shear using a rigid cubical cell. The weaker crushable material also shows markedly less dilation than the stronger crushable material; the weaker sample only just displaying an overall dilation. This makes sense, as when the weaker sample is sheared, to accommodate the macroscopic strain, particles in contact are more likely to break. In the stronger sample (or the unbreakable one), by comparison, the particles are less likely to, or will not fracture and will need to rearrange by sliding and rolling over one another, requiring dilation. Hence for a given initial density, the amount of dilation appears to be linked to the particle strengths. However, as like Bolton et al. [3], strain softening was not clearly observed for the unbreakable simulations. This may indicate that the soils are in fact exhibiting a very wide peak stress, which is supported by the observation that the soils appear as though they are still dilating.

The two breakable materials have different final voids ratios: 0.63 and 0.66 for the weaker and stronger materials respectively. If these are considered to be the critical voids ratios (or approaching such states), then one may conclude that the critical state lines (CSLs) in specific volume-mean stress space are different, and that the position of the CSL appears dependent on the characteristic strength of the particles. This is in accord with the findings in McDowell and de Bono [6], where it was shown that the position of the 

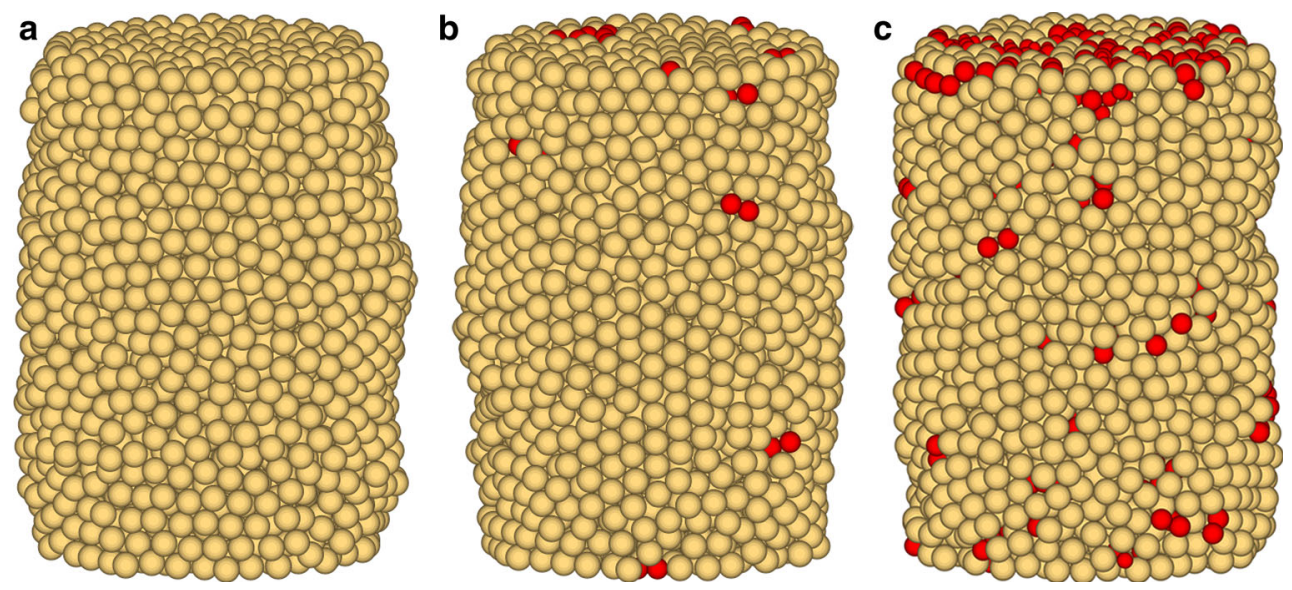

Fig. 3 Images of the final specimens after shearing under $1 \mathrm{MPa}$ of confining pressure to $20 \%$ axial strain: unbreakable simulation (a), crushable simulation with $q_{0}=20 \mathrm{MPa}(\mathbf{b})$, crushable simulation with $q_{0}=10 \mathrm{MPa}(\mathbf{c})$

Fig. 4 Internal view of the crushable simulation with $q_{0}=10 \mathrm{MPa}$, sheared under $1 \mathrm{MPa}$ of confining pressure at 20 axial strain (a), and a histogram showing the vertical distribution of the broken fragments (b)
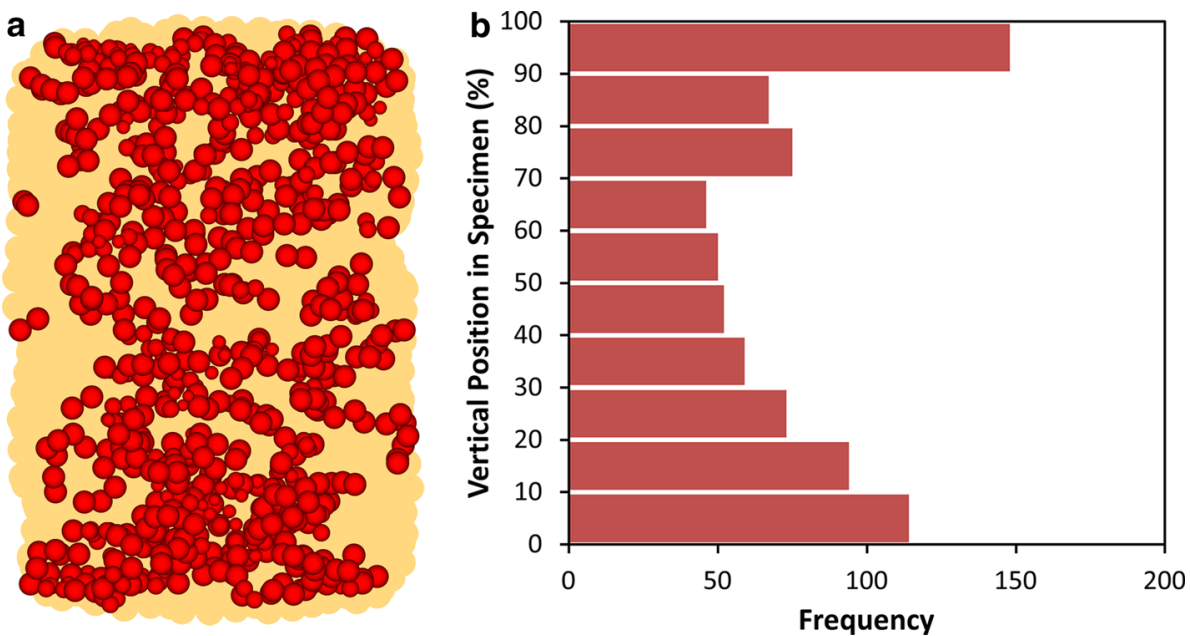

normal compression line (NCL) is related to the average particle strength-as the CSL (in volume-stress space) is often assumed to be parallel with the NCL [20-22]. So one may assume that decreasing the particle strengths changes the position of both the CSL and NCL in volume-stress space.

The stronger crushable material experiences 40 total breakages by an axial strain of $20 \%$, with a fairly constant rate of breakage throughout the test. Of the original 3,350 specimen particles, 3,312 remain at this stage, i.e. 38 have been crushed. The difference in total breakages and the number of original fractured particles is due to the fact that a single particle can break an unlimited number of times. The weaker material by comparison has experienced 463 breakages by the same axial strain, significantly more than the stronger sample. A total of 316 of the original particles have fractured, i.e. $9.43 \%$ by mass; compared to just $1.13 \%$ by mass in the stronger simulation. Despite such a large number of particles undergoing fracture, only seven particles break during isotropic confinement to $1 \mathrm{MPa}$; essentially all breakage occurs during shearing. The three final specimens-with the broken fragments highlighted-are shown in Fig. 3. The material with unbreakable particles is displayed in Fig. 3a. Figure $3 \mathrm{~b}$ shows the stronger crushable sand, and although the figure is an external 3D perspective, several of the broken fragments can be observed across the height of the sample. An equivalent image of the weaker material is shown in Fig. 3c, as well as in Fig. 4a, which gives an internal view of the same sample revealing all broken fragments throughout the depth of the sample. From the latter image, it appears as though there is reduced breakage close to the membrane, and a concentration of breakage close to the platens. This latter observation is confirmed by the histogram given in Fig. 4b, which plots the vertical locations of the fragments as a function of position along the height of the specimen. These two observations are attributable to the fact that if a particle is loaded equally in all directions - e.g. has contacts uniformly spread over its surface-then the particle will have a high mean stress but a low octahedral shear stress; particles that are primarily loaded in one direction - e.g. diametral loadingwill have a high induced shear stress according to Eq. (1). It 
is clear that the particles in contact with either of the platens are unlikely to be surrounded by particles, especially when the neighbouring particles are of the same size. Particles in contact with the membrane on the other hand are more likely to be loaded uniformly, due to the membrane being flexible and consisting of smaller particles, spreading the load.

\section{Confining pressure}

Simulations of crushable sand subjected to triaxial tests with confining pressures up to $4 \mathrm{MPa}$ are now presented. All simulations start with identical specimens, the same as outlined above: the initial voids ratio is 0.75 , the mono-disperse sample consists of spheres of diameter $4 \mathrm{~mm}$, and the initial characteristic particle strength $q_{0}$ is $20.0 \mathrm{MPa}$.

The stress-strain results of four simulations, with confining pressures of 1, 2, 3 and 4MPa are displayed in Fig. 5, in which the simulations reproduce the well-known behaviour of sand in triaxial shearing when subjected to an increase of confining pressure. There is an increase in maximum deviatoric stress, an increase in the axial strain to this stress, and
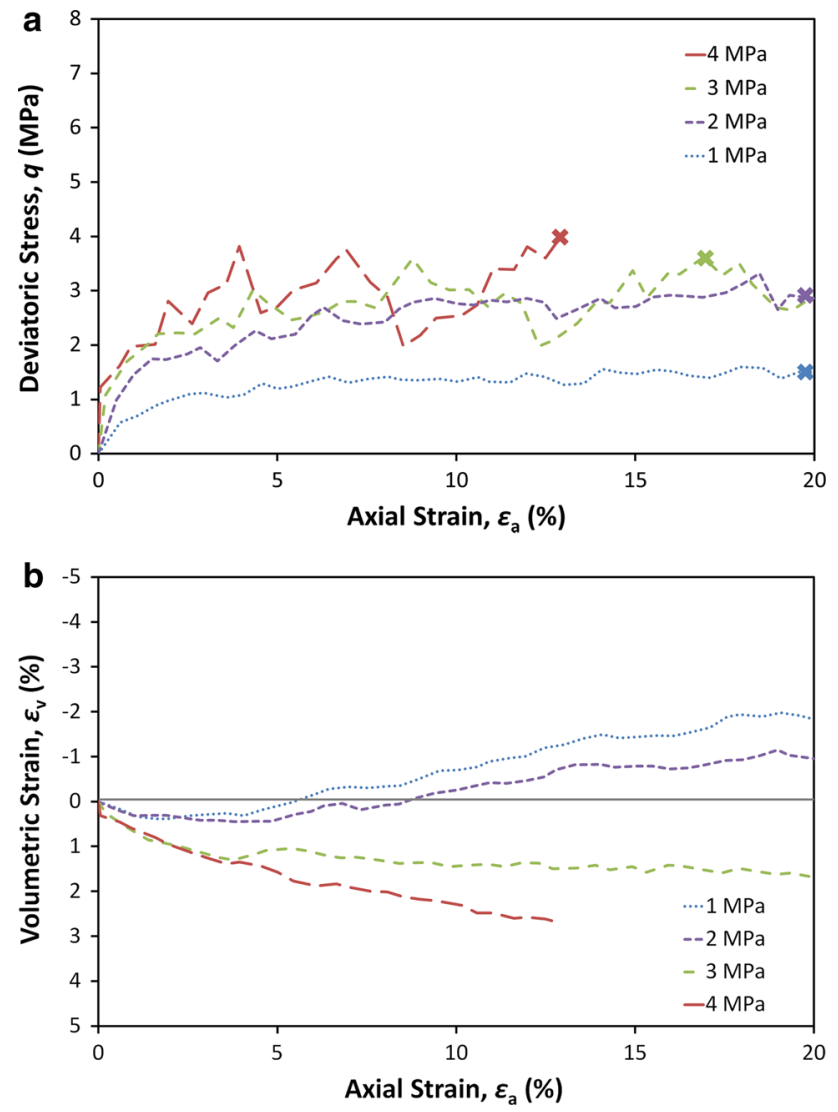

Fig. 5 Triaxial simulation results for crushable sand at various high confining pressures: deviatoric stress response (a) and volumetric response (b) dilation is greatly suppressed causing an overall contraction to be observed. For comparison, an equivalent set of simulations using unbreakable particles are presented in Fig. 6 (the crosses denote the values taken as the ultimate deviatoric stress for each simulation, which will be discussed later). The same pattern of behaviour is observable in the graph giving the deviatoric stress response, although the ultimate values of shear strength appear higher for the unbreakable simulations with $\sigma_{3}=3$ and $4 \mathrm{MPa}$. The most visible difference however is in the volumetric responses; the non-crushable simulations in Fig. 6b reveal very little difference between themselves, with shearing at all levels of confining pressure resulting in dilation. This is due to the inability of the particles to break, and the fact that the samples all assume very similar dense packing upon application of the confining pressure (due to the lack of irregular particle shape).

The difference in ultimate stress displayed by the breakable and unbreakable materials at confining pressures of 3 and $4 \mathrm{MPa}$ could suggest the two materials have different values of the critical state parameter $M$, which would seem contrary to what was said earlier. However, this is the same observation that was made by Bolton et al. [3]. For the sim-
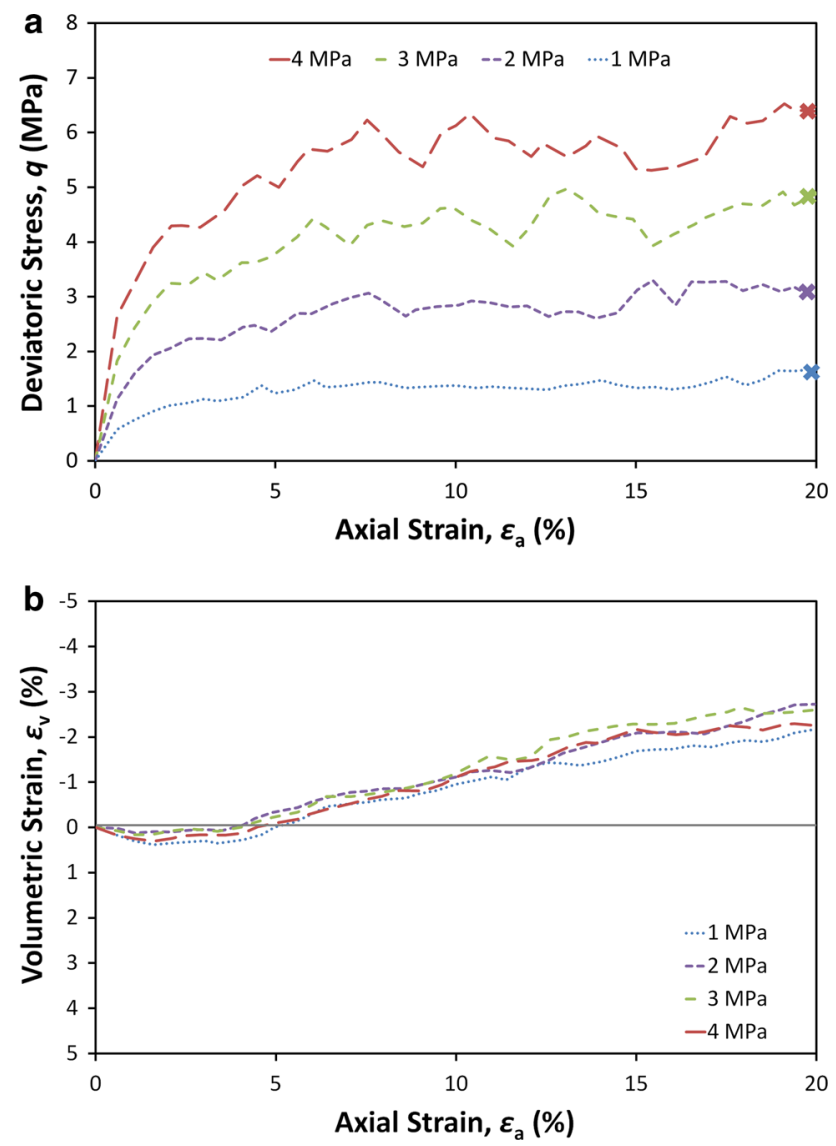

Fig. 6 Triaxial simulation results for sand with unbreakable particles, at various high confining pressures: deviatoric stress response (a) and volumetric response (b) 
ulations with unbreakable particles, although the ultimate stress is proportional to the confining pressure, i.e. $q_{\mathrm{ult}} \propto \sigma_{3}$, they displayed little in terms of post-peak softening, and appear to be still dilating. As suggested earlier, it is possible that further axial strain could reveal a decrease in deviatoric stress $q$, although the lack of strain softening could also be a result of the relatively low number of particles-which may reduce the opportunity for shear bands to develop. Conversely, the breakable materials at $\sigma_{3}=3$ and $4 \mathrm{MPa}$ could still potentially exhibit hardening. In general, Figs. 5 and 6 show the same behaviour as presented in Bolton et al. [3], whose triaxial simulations on unbreakable agglomerates displayed higher ultimate deviatoric stress, and dilation even under confining pressures as high as $40 \mathrm{MPa}$; showing the importance of incorporating particle crushing.

In the simulations using breakable particles, due to the nature of the particle breakage mechanism, there are major fluctuations visible in Fig. 5a, particularly in the simulations with confining pressures of 3 and $4 \mathrm{MPa}$. Due to such a large proportion of particles breaking and being replaced with smaller fragments at any particular occasion, there are noticeable drops in the stress exerted on the platens (and hence the deviatoric stress), which is only recovered after a moderate amount of further axial strain. The simulation with a confining pressure of $4 \mathrm{MPa}$ was terminated at approximately $13 \%$ axial strain; the amount of crushing causing it to become onerous (very small timestep and a large number of different-sized particles).

In the simulations with larger confining pressures ( 3 and $4 \mathrm{MPa}$ ), it may be reasonable to consider the average peak values of deviatoric stress as the ultimate values. The peaks observed in the simulations at 3 and $4 \mathrm{MPa}$ confining pressure are recovered, and appear to be a result of the particle breakage mechanism (as opposed to a single peak resulting from the soil fabric, followed only by strain softening). The breakage mechanism used (Fig. 1) causes a complete loss of contact in the major principal stress direction (due to the placement of the new fragments), which would naturally cause an imminent drop in the global major principal stress. For a dense sample of sand, in reality any particle breakage would not necessarily cause a complete loss of contact, and in any case due to the larger total number of particles and the fact that breakage can occur at any moment in time, the effects on the deviatoric stress would not be as visible. Hence, it can be said that the ultimate stresses increase with increasing confining pressure, with the simulations that were taken to $20 \%$ axial strain displaying fairly constant values. The simulations with $\sigma_{3}=1$ and $2 \mathrm{MPa}$ appear to be approaching and nearly have reached a state of constant volume (indicative of a critical state); the simulation with $\sigma_{3}=3 \mathrm{MPa}$ appears as though it could be still contracting; while the simulation with $4 \mathrm{MPa}$ of confining pressure (which was terminated early), clearly still appears to be contracting.
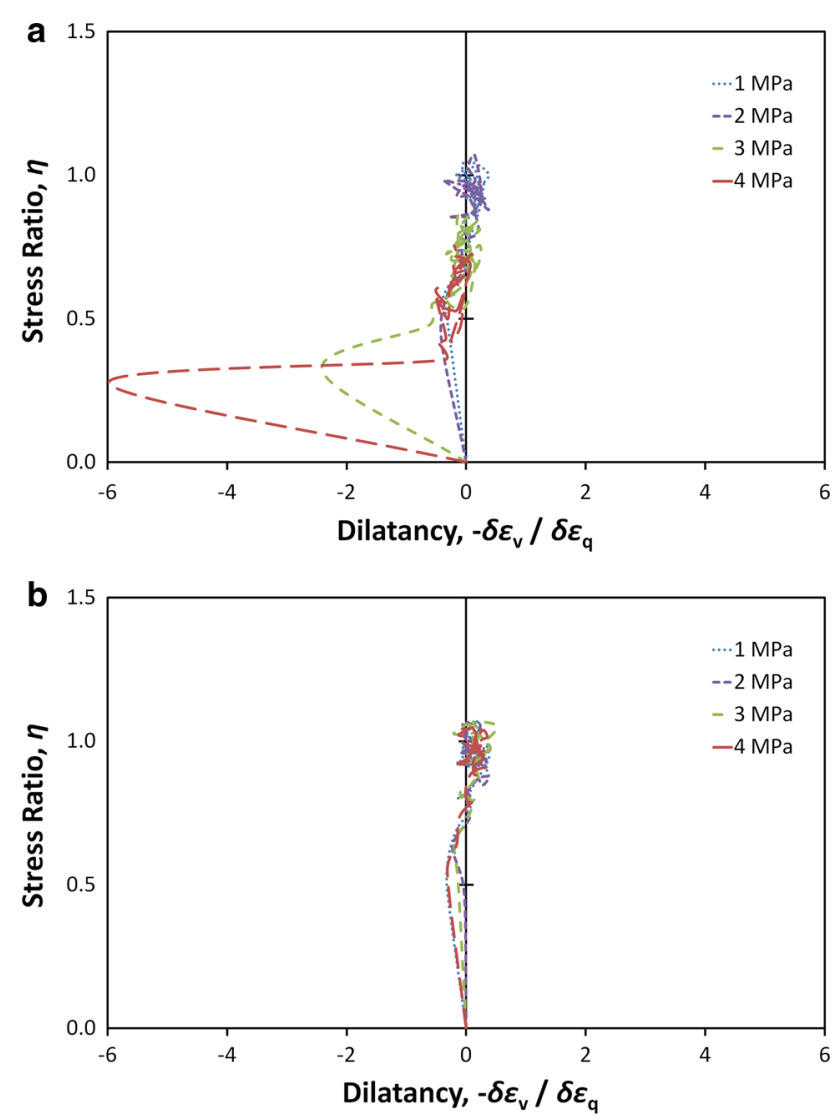

Fig. 7 Stress-dilatancy plots for simulations across a range of confining pressures: crushable sand (a) and non-crushable (b)

The ability of the sand particles to crush enables the simulations at higher confining pressures to exhibit significant contraction, which is not observed in the simulations using unbreakable particles. This more realistic behaviour can also be observed in the stress-dilatancy plots, which are presented in Fig. 7 for both sets of results. Figure 7a shows that the crushable simulations under the highest confining pressure display very little or no positive dilatancy and have lower peak values of dilatancy and stress ratio. Figure $7 \mathrm{~b}$ on the other hand shows that all the non-crushable simulations display behaviour similar to each other, with positive dilatancy exhibited at high levels of confining pressure. The fact that there is positive dilatancy at all levels of confining pressure (and little or no softening) indicates how unrealistic the plastic flow is compared to real sand, confirming the ineffectuality of using unbreakable particles when modelling sand at these pressures.

The stress ratio versus axial strain for the four crushable simulations is shown in Fig. 8. The sand displays slightly lower stress ratios at the higher levels of confinement, however it is possible that continuing the simulations to higher axial strains would result in convergence in the ultimate values of stress ratio, especially when one considers triaxial results from Yamamuro and Lade [2] or Marri et al. [23], in 


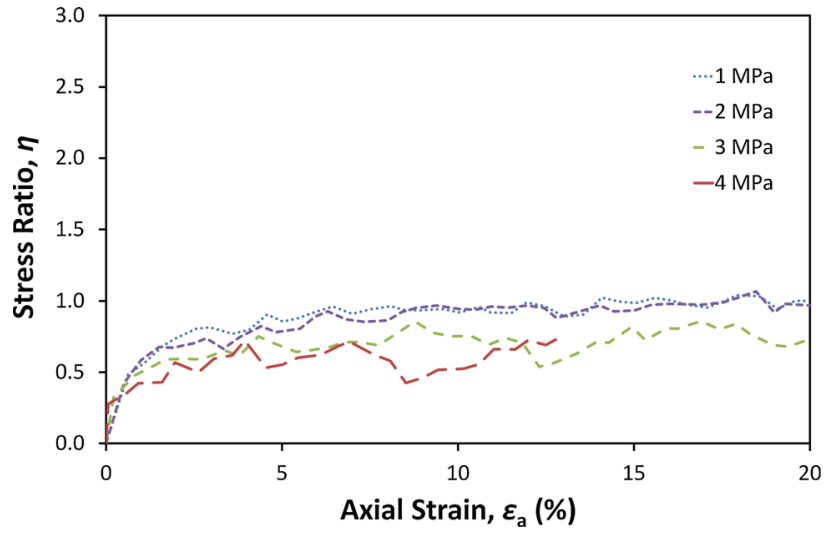

Fig. 8 Graph showing the stress ratio versus axial strain for the four simulations across various high confining pressures

which steady/critical states were only reached at or above $30 \%$ axial strain. If this were to occur, then increasing the confining pressure appears to increase the axial strain at which an apparently steady, ultimate stress is reached. If one overlooks the fluctuations in the deviatoric stress responses for the simulations at 3 and $4 \mathrm{MPa}$, this graph shows a similar trend to results shown by Yamamuro and Lade [2], albeit not across the same range of confining pressures or to the same level of axial strain.

The end point for most of the simulations was $20 \%$ axial strain, however the simulation at the highest confining pressure (4MPa) was terminated prior to this, due to a large number of particles covering a wide range of sizes rendering the calculation time impractical (at the point of termination, the simulation with $4 \mathrm{MPa}$ of confining pressure had undergone over 3,300 breakages, with $d_{\max } / d_{\min }>400$ ). Nonetheless, comparing the state of the simulations at an axial strain of $10 \%$, for example reveals an increasing degree of particle crushing with increasing confining pressure. At this strain, the simulations with $\sigma_{3}=1,2,3$ and $4 \mathrm{MPa}$ had experienced $16,195,677$ and 1,949 breakages respectively. Of the original specimens, 15, 159, 394, and 703 particles had broken, which represent $0.45,5.82,11.76$ and $20.99 \%$ by mass of the original specimens, respectively. There is a clear correlation between level of confining pressure and the degree of crushing exhibited during shear. The key advantage of avoiding the use of agglomerates is that particles can continue to break indefinitely; when using agglomerates, the elementary spheres represent the smallest possible fragments.

Figure 9 plots the overall number of breakages (at 10\% axial strain) as a function of confining pressure, as well as the number of original particles broken. The upper curve shows that the overall number of breakages increases rapidly, and non-linearly with confining pressure. The lower curve however, suggests that the actual number of original particles that break during shearing increases less rapidly (although not linearly) with confining pressure. These two curves together

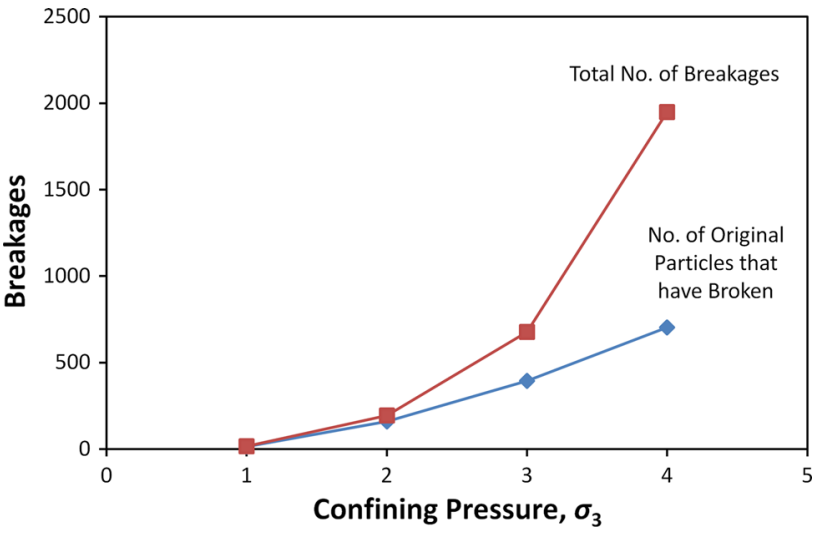

Fig. 9 Graph showing the overall number of breakages in the simulations, and the number of original particles that have fragmented by an axial strain of $10 \%$, as a function of confining pressure

show that at higher pressures, most of the particles that are breaking are themselves fragments of larger broken particles. Yamamuro and Lade [2] presented similar results; they quantified breakage by using Hardin's [1] parameter, and plotted it is a function of the log of $p^{\prime}$ at failure. Their results showed that breakage increases rapidly with increasing pressure, in a similar manner to Fig. 9. Interestingly, their results suggested that breakage slows down, with little increase in breakage observed in tests with $\sigma_{3}>20 \mathrm{MPa}$.

The observation that it is the smaller particles-i.e. broken fragments themselves-that continue to break agrees with previous studies on the evolution of fractal particle size distributions. McDowell and Bolton [17] for example discussed how the probability of fracture for a particle is determined not only by the applied stress and particle size, but also the coordination number. McDowell and de Bono [6] analysed the contact distribution of their normally-compressed sample of sand, and found that the total number of contacts reduced with particle size-i.e. the largest particles were surrounded by many smaller-sized particles and had a large total number of contacts; the smallest particles by comparison had far fewer contacts, as it was not possible for these to have lots of smaller particles surrounding them. Hence smaller particles are more likely to be loaded diametrally - and therefore be under a larger octahedral shear stress.

Figure 10 shows the total number of particles as a function of axial strain, for the four simulations at different confining pressures. The graph substantiates the observation from the previous figure that confining pressure greatly increases the degree of crushing. The trends of the curves in Fig. 10, particularly for the simulations at 3 and $4 \mathrm{MPa}$ confining pressures, demonstrate that breakage increases rapidly with shearing, and that breakage shows no indication of reducing as steady states are approached. However, it appears that most of breakages that continue to occur are the fragments, which comprise the smallest particles; this would suggest that the particle size 


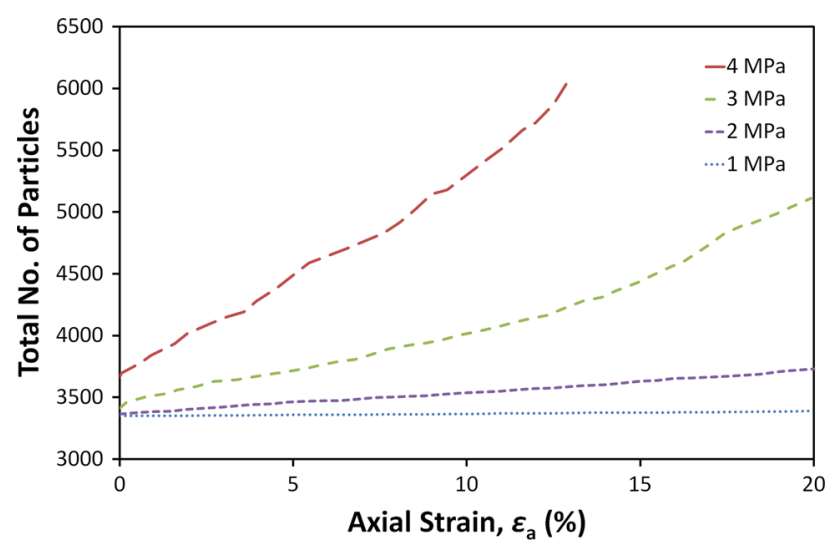

Fig. 10 Graph showing the total number of particles, as a function of axial strain for the four simulations at various confining pressures

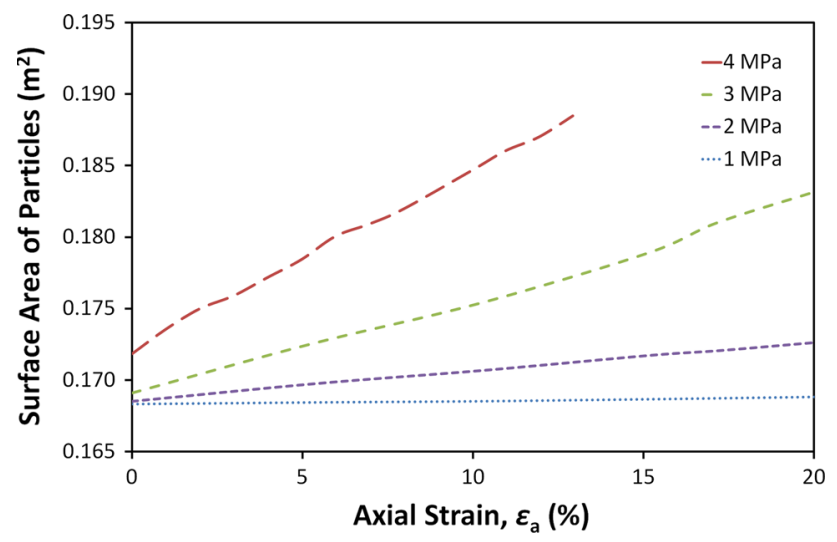

Fig. 11 Graph showing the total surface area of the particles as a function of axial strain for the four simulations at various confining pressures

distributions are still evolving, potentially tending to fractal distributions.

Figure 11 displays the total surface area of the particles as a function of axial strain. The curves in this figure appear to increase more linearly than those in Fig. 10, although they also show no indication of reaching ultimate values. This suggests that there is still significant crushing occurring when the simulations were terminated, which one may not expect at critical states, when the volume is constant. Miura and Yamamoto [24] and Miura and O-hara [25]—who quantified breakage during triaxial shearing by measuring the change in surface area-showed that the particle surface area was still increasing at axial strains as high as $50 \%$; when the deviatoric stress and volumetric strain appeared to be reaching stable values. Chandler [26], who distinguished grain damage and rearrangement as separate mechanisms, perceived the critical state as the point at which the volume changes due to these competing mechanisms cancel out. Additionally, Coop et al. [27] also conducted a series of ring shear tests, and showed that not only particle crushing, but also volumetric strains continue to occur at very high strains. Although the rate of change of volume was small, Coop et al. [27] showed that in most cases the volumetric strain only completely stabilised at shear strains of around $2,000 \%$. Hence this would suggest that crushing continues to very high strains during shear, and that the volume also may continue to change, although by a very small amount.

Although crushing is still clearly occurring in the simulations, it is largely the smaller particles that are continuing to fragment; in reality, it is likely the smallest particles would eventually stop breaking due to a comminution limit, leading to an ultimate particle size distribution, which was also suggested by Coop et al. [27].

Inner views of the samples showing the broken fragments are given in Fig. 12, which reveal the increasing degree of crushing experienced by the four simulations. The images are taken at $10 \%$ axial strain, and all the crushed fragments are highlighted, throughout the depths of the samples. Figure $12 \mathrm{~d}$, which shows that breakage has occurred extensively, throughout most of the specimen, shows that the smallest fragments are located around the central z-axis, again indicating that particles on the outside of the specimen are less likely to break.

For the simulations at 1 and $2 \mathrm{MPa}$ of confining pressure, the ultimate deviatoric stress also constitutes the peak (failure) stress. For the two simulations with higher confining pressures, it is the average peak values in Fig. 5 which are considered the ultimate deviatoric stress in the following analysis (this is because the stress troughs are caused by particle breakage, and are recovered; as opposed to a single peak resulting from the soil packing). Although Fig. 5 suggests that the breakable soils haven't yet all reached critical states, the ultimate states are plotted in deviatoric stress-mean effective stress space in Fig. 13a, alongside the equivalent simulations using unbreakable particles (the values taken as the ultimate deviatoric stress, $q_{\mathrm{ult}}$, are marked on the stress-strain plots in Figs. 5, 6). Figure 13b shows the ultimate states for the two sands plotted on the conventional specific volume-mean stress space.

The disparity between the crushable and non-crushable simulations in Fig. 13a at confining pressures of 3 and $4 \mathrm{MPa}$ could be interpreted as indicating different CSLs, and hence different values of $M$ for the two materials; with the noncrushable material having a slightly higher value (approximately equal to 1 ). The points from the crushable simulations give lower ultimate values of deviatoric stress, $q$, and indicate a curve - the angle of friction appearing to decrease with confining pressure. Although only four points are plotted for each material, and that these states cannot be considered to be truly critical, similar observations were made by Cheng et al. [8], who conducted constant $p^{\prime}$ shear tests using agglomerates in a cubical cell. The maximum angle of internal friction for their agglomerates continued to decrease with the logarithm of mean stress, apparently approaching the angle of inter-particle friction, even after deviatoric strains 
Fig. 12 Inner views of specimens after shearing to $10 \%$ axial strain, showing highlighted broken fragments from simulations across a range of confining pressures: $1 \mathrm{MPa}(\mathbf{a})$, $2 \mathrm{MPa}(\mathbf{b}), 3 \mathrm{MPa}(\mathbf{c})$ and $4 \mathrm{MPa}$ (d) a

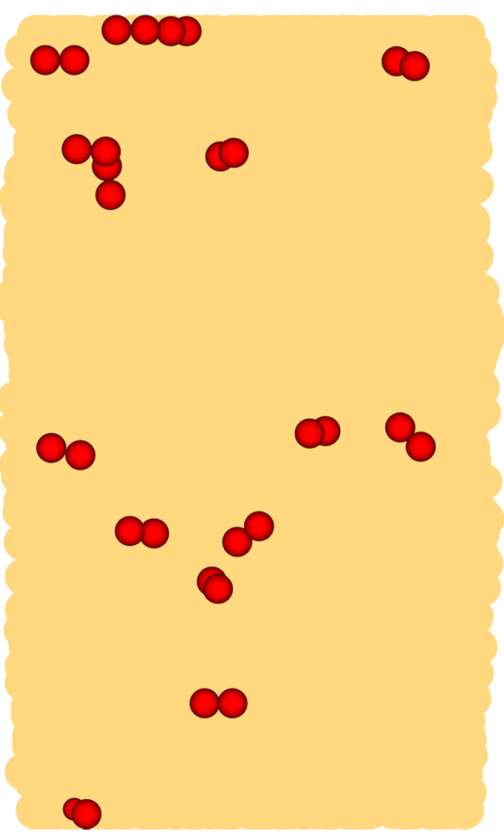

C

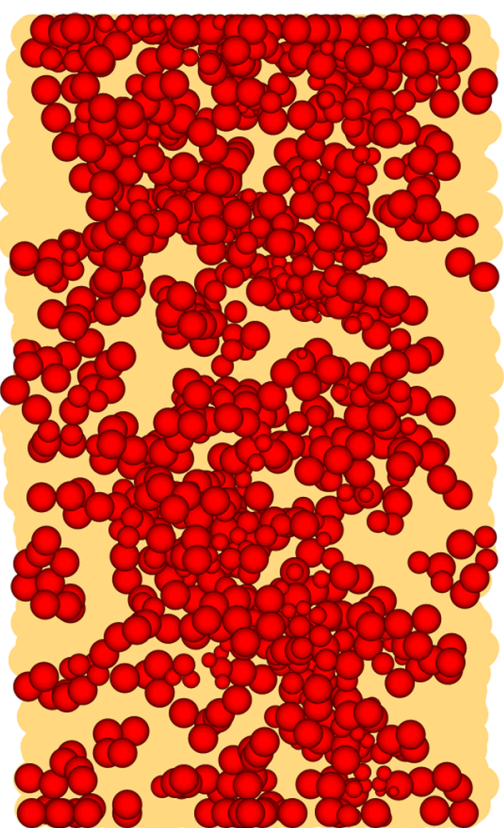

b

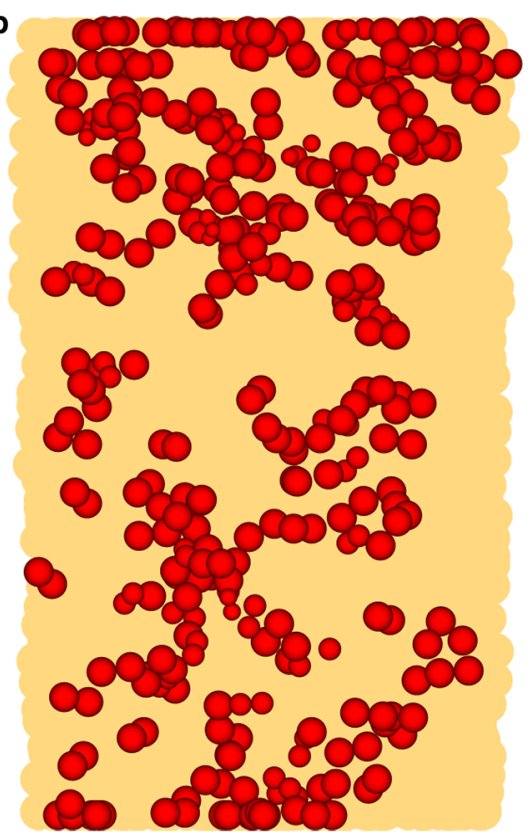

d

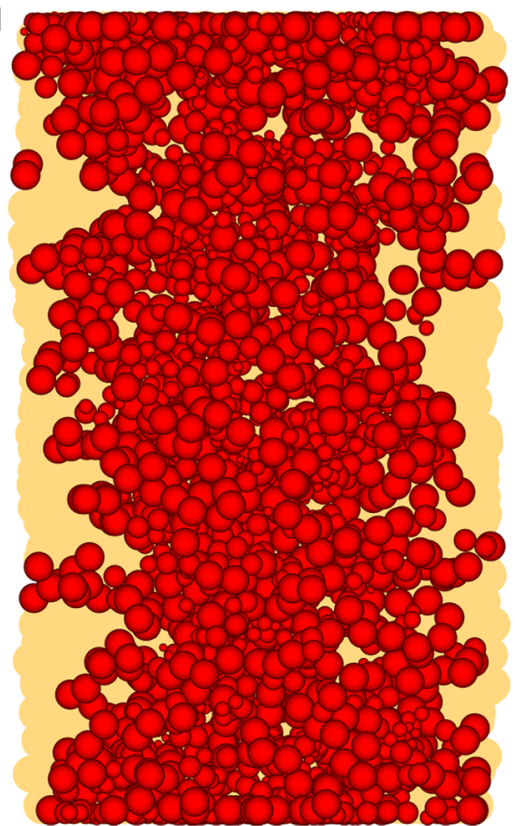

of $40 \%$. However, as discussed earlier with regard to the crushable simulations presented here, the shear tests conducted at confining pressures of 1 and $2 \mathrm{MPa}$ were unable to exhibit any softening, and the simulation at a confining pressure of $4 \mathrm{MPa}$ was likely to still exhibit hardening. This indicates that the friction angles mobilised in these simulations are not representative of the critical state friction angle of the soil. It is worth noting that if particle shape was incorporated, much steeper, more realistic CSLs would be achieved in $q-p^{\prime}$ space. In Fig. 13b, the two sets of points are distinct from one another, although neither suggest completely linear CSLs in specific volume-mean stress space. However, if comparison is drawn between the two sets of simulations, one can see that the points for the crushable simulation indicate a CSL that has a noticeably larger negative gradient-i.e. steeper. Although there are too few points to fit a trendline, it is evident that the crushable material appears to offer much more realistic behaviour compared to the non-crushable simulations, when one considers the typical gradient for CSLs observed for sands across (e.g. [28-30]).

These states for the crushable sand are plotted in voids ratio-mean effective stress space in Fig. 14, with doublelogarithmic axes. McDowell and de Bono [6] showed that the slope of the NCL, i.e. the compressibility index $C_{\mathrm{c}}$, when plotted on $\log e-\log \sigma_{\mathrm{v}}$ space is given by $C_{c}=1 / 2 b$, where 

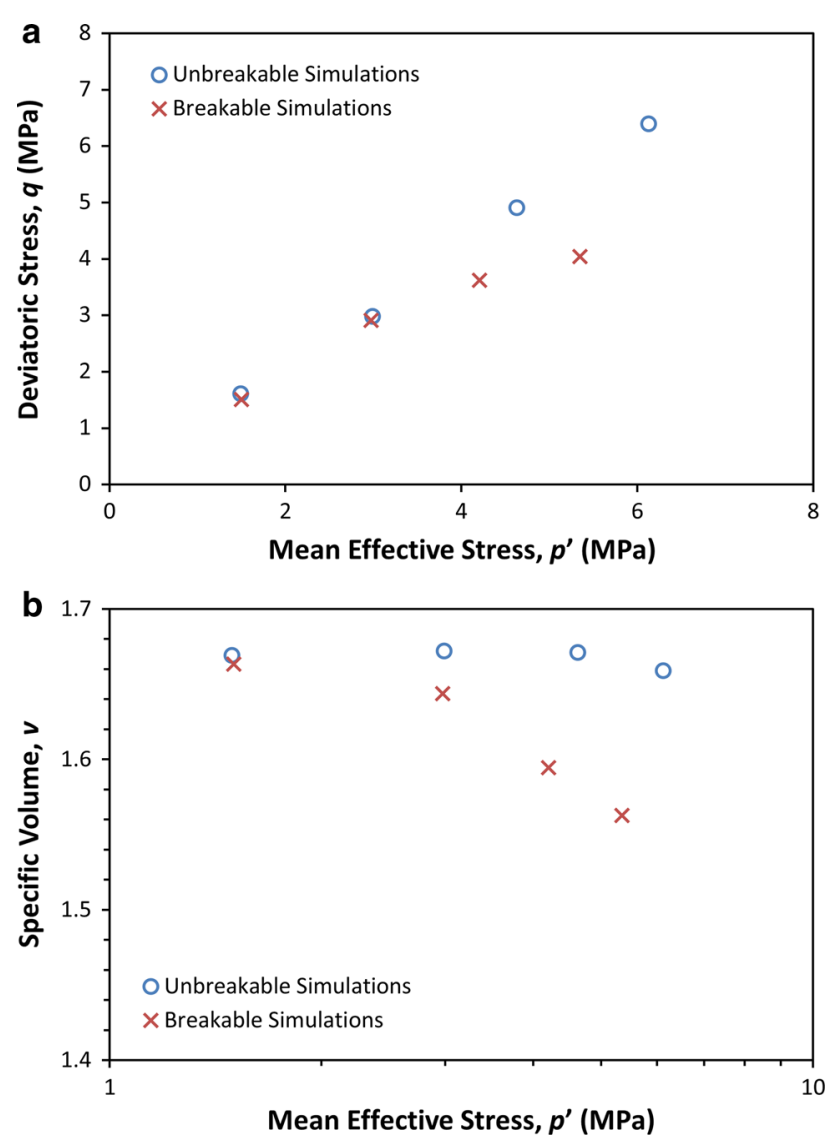

Fig. 13 Critical/ultimate states of simulations on crushable and noncrushable sand under a range of confining pressures, plotted in $q-p^{\prime}$ space (a) and $v-\log p^{\prime}$ space (b)

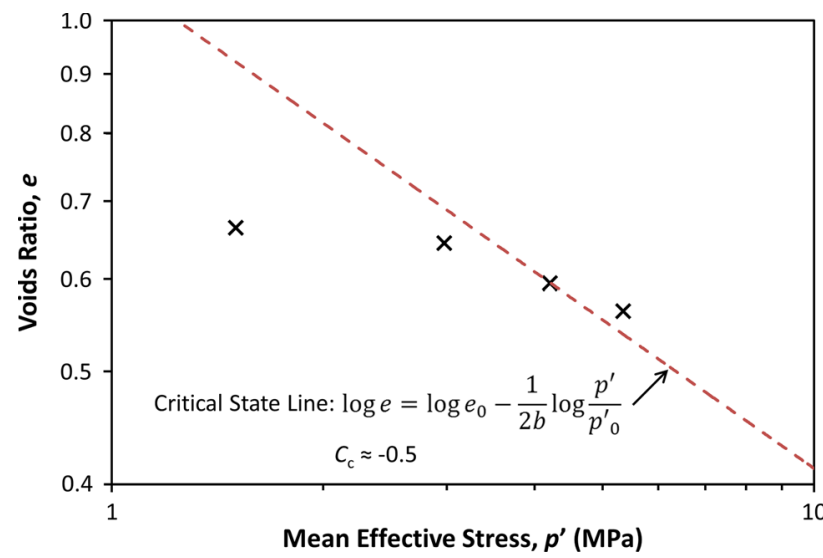

Fig. 14 Critical/ultimate states from simulations of unbonded, crushable sand and idealised CSL plotted in $e-p^{\prime}$ space

$b$ is the size-hardening effect from Eq. (3). The NCL also had this slope when plotted in $\log \mathrm{e}-\log p^{\prime}$ space [31]. Hence if the CSL is indeed parallel with the NCL, then as proposed by McDowell and de Bono [6] the slope of the CSL will be linear when plotted in double-logarithmic voids ratio-mean stress space, and may be described by: $\log e=\log e_{0}-\frac{1}{2 b} \log \frac{p^{\prime}}{p_{0}^{\prime}}$

where $e$ is the voids ratio, $e_{0}$ and $p_{0}^{\prime}$ define a reference point on the CSL, and $b$ is the size-hardening law from Eq. (3), and controls the slope of the NCL-i.e. the rate at which the volume of voids decreases as the logarithm of mean effective stress increases.

For the material used in these simulations, $b=(3 / 3.3)$, so the slope $(=1 / 2 b)$ is approximately 0.5 . An idealised CSL with this slope is also shown in Fig. 14. At low mean effective stresses, critical state points in $e-p^{\prime}$ or $v-p^{\prime}$ space often do not conform to the CSL at high stresses; i.e. if the linear CSL at high stresses is extrapolated, at low stresses critical state points usually lie below this line $[32,33]$. Considering that the rightmost point is still contracting and moving downwards (Fig. 5) towards the idealised CSL, the points are encouraging, and appear to support a CSL that is parallel with the $\mathrm{NCL}$, and linear in $\log e-\log p^{\prime}$ space, although further points at higher pressures are desired.

\section{Conclusions}

The series of simulations presented in this paper has demonstrated that it is possible to simulate high-pressure triaxial tests on a crushable soil, with a large number of particles, using a flexible membrane that allows free deformation. Sand was modelled using an aggregate of crushable particles, in which the octahedral shear stress was used as the fracture criterion. Significantly, it was shown that allowing particles to break enables realistic volumetric strain to be observed, with the crushable materials able to exhibit overall contraction instead of dilation. This is similar to the observations by Bolton et al. [3], but with a larger number of particles, and realistic laboratory-style triaxial conditions.

Using a large number of particles (with the ability to crush an unlimited number of times) allowed the distribution of fragments to be explored; breakage appeared concentrated around the platens and around the central vertical axis of the specimen. The particle strengths had a major influence on the degree of crushing, which in turn influenced the amount of contraction.

The confining pressure was shown to increase the quantity of breakage (Fig. 9), and in the simulations at higher confining pressures, it was observed that it was the breaking of the fragments themselves that accounted for the majority of overall breakages; suggesting that as breakage continues during shear, a range of particle sizes develops. The simulations were still exhibiting breakage at axial strains of $20 \%$; however, although the overall number of particles appeared to be increasing rapidly, by monitoring the change in surface area it appeared as though it was the smallest particles that were continuing to break. This could indicate the eventual 
emergence of fractal particle size distributions, and explain why the rate of volume change with axial strain decreases despite continued crushing. Future work will aim towards conducting triaxial simulations with a larger number of initial particles, and/or to further axial strains, to improve realism. Although the presented simulations at higher confining pressures comprised reasonable quantities of particles after undergoing breakage, these quantities were not on the scale of experimental triaxial tests; such relatively small numbers of sand particles may suppress localisation. With increased computing power, additional further work would include repeating such simulations numerous times to obtain more reliable results. Although clear trends were observed, each simulation only represented a single realisation for each set of variables.

From the results of simulations under a range of confining pressures, using the strength characteristics of a silica sand, the critical states were investigated. The ultimate states from the simulations were plotted in deviatoric-mean stress space and voids ratio-mean stress space (as well as the conventional specific volume-mean stress space). According to the hypothesis put forward by McDowell and de Bono [6]which stated that the slope of the NCL is a function of the size-hardening law - an idealised CSL with this theoretical slope was also plotted in voids ratio-mean stress space, under the assumption that the CSL and NCL are parallel at high stresses-once a significant number of particles have resulted from crushing. The results were encouraging, and points from the simulations with the highest confining pressures appeared to be approaching this theoretical CSL; however, further tests at much higher confining pressures are needed to fully define the CSL in three-dimensional volume-stress space.

Acknowledgments The authors are grateful to the Engineering and Physical Sciences Research Council (EPSRC) for their financial support through the Doctoral Training fund.

\section{References}

1. Hardin, B.O.: Crushing of soil particles. J. Geotech. Eng. 111, 1177-1192 (1985)

2. Yamamuro, J.A., Lade, P.V.: Drained sand behavior in axisymmetric tests at high pressures. J. Geotech. Eng. 122, 109-119 (1996)

3. Bolton, M.D., Nakata, Y., Cheng, Y.P.: Micro- and macromechanical behaviour of DEM crushable materials. Géotechnique 58, 471-480 (2008)

4. Lim, W.L., McDowell, G.R.: The importance of coordination number in using agglomerates to simulate crushable particles in the discrete element method. Géotechnique 57, 701-705 (2007)

5. De Bono, J., McDowell, G., Wanatowski, D.: Discrete element modelling of a flexible membrane for triaxial testing of granular material at high pressures. Géotech. Lett. 2, 199-203 (2012)

6. McDowell, G.R., de Bono, J.P.: On the micro mechanics of one-dimensional normal compression. Géotechnique 63, 895-908 (2013)

7. Itasca: Particle Flow Code in 3 Dimensions. Itasca Consulting Group Inc, Minneapolis, Minnesota (2005)
8. Cheng, Y.P., Bolton, M.D., Nakata, Y.: Crushing and plastic deformation of soils simulated using DEM. Géotechnique 54, 131-141 (2004)

9. Åström, J.a, Herrmann, H.J.: Fragmentation of grains in a twodimensional packing. Eur. Phys. J. B Condens. Matter Complex Syst. 5, 551-554 (1998)

10. Tsoungui, O., Vallet, D., Charmet, J.: Numerical model of crushing of grains inside two-dimensional granular materials. Powder Technol. 105, 190-198 (1999)

11. Lobo-Guerrero, S., Vallejo, L.E.: Crushing a weak granular material: experimental numerical analyses. Géotechnique 55, 245-249 (2005)

12. Ben-Nun, O., Einav, I.: The role of self-organization during confined comminution of granular materials. Philos. Trans. R. Soc. A Math. Phys. Eng. Sci. 368, 231-247 (2010)

13. Ben-Nun, O., Einav, I., Tordesillas, a: Force attractor in confined comminution of granular materials. Phys. Rev. Lett. 104, 108001 (2010)

14. McDowell, G.R., Harireche, O.: Discrete element modelling of soil particle fracture. Géotechnique 52, 131-135 (2002)

15. Harireche, O., Mcdowell, G.R.: Discrete element modelling of yielding and normal compression of sand. Géotechnique 52, 299$304(2002)$

16. Jaeger, J.C.: Failure of rocks under tensile conditions. Int. J. Rock Mech. Min. Sci. Geomech. Abstr. 4, 219-227 (1967)

17. McDowell, G.R., Bolton, M.D.: On the micromechanics of crushable aggregates. Géotechnique 48, 667-679 (1998)

18. McDowell, G.R., Amon, A.: The application of weibull statistics to the fracture of soil particles. Soils Found. 40, 133-141 (2000)

19. McDowell, G.R.: On the yielding and plastic compression of sand. Soils Found. 42, 139-145 (2002)

20. Bolton, M.D.: A Guide to Soil Mechanics. Macmillan Press, London (1979)

21. Coop, M.R.: The mechanics of uncemented carbonate sands. Géotechnique 40, 607-626 (1990)

22. Craig, R.F.: Soil Mechanics. Taylor and Francis, New York (2004)

23. Marri, A., Wanatowski, D., Yu, H.S.: Drained behaviour of cemented sand in high pressure triaxial compression tests. Geomech. Geoengin. 7, 159-174 (2012)

24. Miura, N., Yamamoto, T.: Particle crushing properties of sands under high stresses. Technology reports of the Yamaguchi University 1(4), 439-447 (1976)

25. Miura, N., O-hara, S.: Particle-crushing of a decomposed granite soil under shear stresses. Soils Found. 19, 1-14 (1979)

26. Chandler, H.W.: A plasticity theory without drucker's postulate, suitable for granular materials. J. Mech. Phys. Solids 33, 215-226 (1985)

27. Coop, M.R., Sorensen, K.K., Bodas Freitas, T.: Particle breakage during shearing of a carbonate sand. Géotechnique 54, 157-163 (2004)

28. Wang, J.: The stress-strain characteristics of Portaway Sand. Ph.D. thesis, University of Nottingham, Nottingham, UK (2005)

29. Wanatowski, D., Chu, J.: Drained behaviour of Changi sand in triaxial and plane-strain compression. Geomech. Geoengin. 2, 2939 (2007)

30. Marri, A.: The mechanical behaviour of cemented granular material at high pressures. Ph.D. thesis, University of Nottingham, Nottingham, UK (2010)

31. McDowell, G.R., de Bono, J.P., Yue, P., Yu, H.-S.: Micro mechanics of isotropic normal compression. Géotech. Lett. 3, 166-172 (2013)

32. Wood, D.M.: Soil Behaviour and Critical State Soil Mechanics. Cambridge University Press, Cambridge (1990)

33. Russell, A.R., Khalili, N.: A bounding surface plasticity model for sands exhibiting particle crushing. Can. Geotech. J. 41, 1179-1192 (2004) 